Illinois State University

ISU ReD: Research and eData

Theses and Dissertations

$1-18-2014$

\title{
'i Like You Both The Same, But For Different Reasons': Differences Between Communication Mediums Related To Self-Disclosure, Responsiveness, And Self-Awareness
}

Kevin Joseph Wallpe

Illinois State University, kjwallpe@gmail.com

Follow this and additional works at: https://ir.library.illinoisstate.edu/etd

Part of the Communication Commons, and the Social Psychology Commons

\section{Recommended Citation}

Wallpe, Kevin Joseph, "'i Like You Both The Same, But For Different Reasons': Differences Between Communication Mediums Related To Self-Disclosure, Responsiveness, And Self-Awareness" (2014). Theses and Dissertations. 105.

https://ir.library.illinoisstate.edu/etd/105

This Thesis is brought to you for free and open access by ISU ReD: Research and eData. It has been accepted for inclusion in Theses and Dissertations by an authorized administrator of ISU ReD: Research and eData. For more information, please contact ISUReD@ilstu.edu. 


\section{'I LIKE YOU BOTH THE SAME, BUT FOR DIFFERENT REASONS': DIFFERENCES BETWEEN COMMUNICATION MEDIUMS \\ RELATED TO SELF-DISCLOSURE, RESPONSIVENESS, AND \\ SELF-AWARENESS}

Kevin J. Wallpe

68 Pages

May 2014

As computer-mediated communication (CMC) increasingly becomes a part of people's everyday lives (Mesch \& Talmud, 2006), it becomes important to understand what differentiates this mode of communication from traditional face-to-face $(\mathrm{FtF})$ communication. Some studies have shown that $\mathrm{CMC}$ interactions are related to greater liking (relative to FtF) of previously unacquainted interaction partners (McKenna, Green, \& Gleason, 2002; Ramirez \& Zhang, 2007), whereas others have concluded just the opposite -- that FtF interactions are related to more liking of previously unacquainted interaction partners (Mallen, Day, \& Green, 2003; Okdie, Guadagno, Bernieri, Geers, \& Mclarney-Vesotski, 2011). What leads to the inconsistent findings concerning the effect of communication medium on reported liking? The current research examined liking, perceived self-disclosure, perceived partner responsiveness, and state self-awareness across different communication mediums. 
Unacquainted pairs of participants interacted with one another in different communication mediums. Participants were instructed to "get acquainted" with one another through an unstructured 20-minute interaction. Participants were randomly assigned to interact with one another through a text-only $\mathrm{CMC}$, an audio/video CMC, or a FtF communication medium. Following the interaction, participants completed measures regarding liking of their interaction partner, perceived own self-disclosure, perceived partner self-disclosure, perceived partner responsiveness, and state self-awareness. Significant multivariate results emerged; with differences in self-disclosure, responsiveness, and self-awareness based on the communication mediums with $\mathrm{FtF}$ and audio/video CMC scoring significantly higher than text-only CMC. These results revealed that participants reported liking their interaction partners significantly more when interacting via FtF or audio/video CMC compared to text-only CMC. Levels of disclosure and responsiveness significantly predicted liking for one's interaction partner. Methodological and theoretical implications are discussed 
'I LIKE YOU BOTH THE SAME, BUT FOR DIFFERENT REASONS': DIFFERENCES BETWEEN COMMUNICATION MEDIUMS

RELATED TO SELF-DISCLOSURE,

RESPONSIVENESS, AND

SELF-AWARENESS

KEVIN J. WALLPE

A Thesis Submitted in Partial Fulfillment of the Requirements for the Degree of

MASTER OF SCIENCE

Department of Psychology

ILLINOIS STATE UNIVERSITY

2014 
C 2014 Kevin J. Wallpe 
'I LIKE YOU BOTH THE SAME, BUT FOR DIFFERENT REASONS': DIFFERENCES BETWEEN COMMUNICATION MEDIUMS

RELATED TO SELF-DISCLOSURE, RESPONSIVENESS, AND

SELF-AWARENESS

KEVIN J. WALLPE

COMMITTEE MEMBERS:

Susan Sprecher, Chair

Joel Schneider

Marla Reese-Weber 


\section{ACKNOWLEDGMENTS}

I would like to thank my committee, Drs. Susan Sprecher, Joel Schneider, and Marla Reese-Weber, who were generous with their time and discerning in their reading and guidance. The GTA provided by ISU helped me fund my endeavors. I would also like to thank my research assistants: Michelle Duda, Emily Gannon, Samantha Helms, Christina Mills, and Miranda H. They contributed a tremendous amount of time and efforts helping me collect the data used in the current research.

K.J.W. 


\section{CONTENTS}

Page

ACKNOWLEGMENTS $\quad$ i

CONTENTS

TABLES

CHAPTER

I. INTRODUCTION 1

Statement of the Problem 1

II. REVIEW OF RELATED LITERATURE 3

General Literature Review 3

Early CMC Research $\quad 3$

Recent CMC Research 10

Self-Disclosure 14

Responsiveness 18

Self-Consciousness and Self-Awareness $\quad 21$

$\begin{array}{ll}\text { The Current Research } & 25\end{array}$

$\begin{array}{ll}\text { III. METHOD } & 31\end{array}$

Participants $\quad 31$

$\begin{array}{ll}\text { Materials } & 32\end{array}$

Procedure $\quad 32$

Pre-Interaction Measures 33 
Post-Interaction Measures

$\begin{array}{ll}\text { IV. RESULTS } & 36\end{array}$

$\begin{array}{ll}\text { Results } & 36\end{array}$

Descriptive Statistics $\quad 36$

Tests of Hypotheses $\quad 37$

Tests of Research Questions $\quad 38$

Liking $\quad 39$

Topics of Disclosure $\quad 40$

Difficulty of Communication Medium 40

$\begin{array}{ll}\text { V. DISCUSSION } & 42\end{array}$

General Discussion $\quad 42$

Methodological Issues $\quad 43$

Hypotheses $\quad 43$

Research Questions $\quad 46$

Implications $\quad 48$

Communication Medium $\quad 48$

Methodological Implications $\quad 49$

Theoretical Implications $\quad 52$

Strengths and Limitations $\quad 53$

Future Directions $\quad 55$

Conclusion $\quad 57$

$\begin{array}{ll}\text { REFERENCES } & 58\end{array}$ 


\section{TABLES}

Table $\quad$ Page

1. Descriptive Statistics and Correlations Among Dependent Measures 63

2. Means, Standard Deviations, and Effect Sizes for Perceived Self-Disclosure, Perceived Partner Self-Disclosure, Perceived Partner Responsiveness, and Private Self-Awareness

3. Multiple Regression on Liking with Self-Disclosure, Partner Disclosure, and Responsiveness as Predictors

4. Multiple Regression on Liking with Self-Disclosure, Partner Disclosure, and Responsiveness as Predictors for FtF, Audio/Video, and Text-Only Conditions

5. Means and Standard Deviations for Disclosure Topics

6. Means and Standard Deviations for Reported Difficulty in Self-Disclosure and Responsiveness 


\section{CHAPTER I \\ INTRODUCTION}

\section{Statement of the Problem}

As computer-mediated communication (CMC) increasingly becomes a part of people's everyday lives (Mesch \& Talmud, 2006), it becomes important to understand what differentiates this mode of communication from traditional face-to-face $(\mathrm{FtF})$ communication. Some studies have shown that CMC interactions are related to more liking (than FtF) between previously unacquainted interaction partners (McKenna, Green, \& Gleason, 2002; Ramirez \& Zhang, 2007), whereas others have concluded just the opposite -- that FtF interactions are related to more liking between previously unacquainted interaction partners (Mallen, Day, \& Green, 2003; Okdie, Guadagno, Bernieri, Geers, \& Mclarney-Vesotski, 2011). What leads to the inconsistent findings concerning the effect of communication medium on reported liking? In the current research, I addressed this question by having unacquainted pairs of participants interact in different communication mediums. After their interaction, the participants completed measures of liking, perceived own self-disclosure, perceived partner self-disclosure, and perceived partner responsiveness (Collins \& Miller, 1994; Greene, Derlega, \& Mathews, 2006; Reis, Clark, \& Holmes, 2004). I also included measures of trait self-consciousness as an individual difference variable prior to the interaction and state self-awareness 
following the interaction, which have been found to be related to levels of self-disclosure (Davis \& Franzoi, 1987) and may moderate the influence of communication medium on liking and other related variables including perceived partner responsiveness (Matheson \& Zanna, 1988).

I will begin by reviewing the literature on $\mathrm{CMC}$ in chronological order, first presenting the earliest research conducted on the effects of CMC. Following this review, a focus will be given to research on self-disclosure and perceived partner responsiveness. The literature review will conclude by giving an overview of self-consciousness and selfawareness, and particularly how they may moderate the influence of communication medium on self-disclosure and perceived partner responsiveness. 


\section{CHAPTER II}

\section{REVIEW OF RELATED LITERATURE}

\section{General Literature Review}

\section{Early CMC Research}

The early environment of CMC throughout the 1990's and early 2000's involved largely text-based communications; and therefore reduced visual, auditory, and contextual cues compared to traditional modes of communication such as $\mathrm{FtF}$ and the telephone (Parks \& Floyd, 1996). CMC during the 1990's and early 2000's lacked many important aspects emphasized in traditional relationship development; such as physical proximity, frequent interaction, information about physical features, cues about group membership, and information about the broader social context (Parks \& Floyd, 1996). The early research on CMC focused on the lack of cues (e.g. nonverbal, contextual) being the essential difference between traditional FtF interactions and $\mathrm{CMC}$ interactions. The lack of cues within the CMC environment lead to the speculation of an impoverished social experience (Bargh \& McKenna, 2004) filled with cold, impersonal, and unsociable interactions (Sherman, 2003). In FtF interactions, context cues may include the environment, gestures/facial features, symbols of authority and status, physical appearance, and spatial behavior. Within CMC, these cues are largely absent when using text-only communication (Tidwell \& Walther, 2002). 
During the 1990's, the question of whether relationships can be formed through CMC was beginning to be investigated. Parks and Floyd (1996) were among the first researchers to examine whether social relationships are formed online. They administered surveys to randomly selected newsgroup members regarding relationship formation. The results showed that $61 \%$ of respondents had indeed formed a relationship through the newsgroup, and that $30 \%$ reported communicating with these individuals at least three or four times a week. In addition to forming these relationships online, many respondents reported employing other mediums of communication supplemented to their newsgroup participation; with $35 \%$ reporting telephone contact, $28 \%$ postal service, and $33 \%$ reporting meeting FtF with their online acquaintances. Relationships formed online do not appear to remain there for long, with $64 \%$ of the participants in the Parks and Floyd (1996) study reporting the use of communication channels other than the computer. Parks and Floyd (1996) concluded that relationships appear to form online and that the best predictors of who formed relationships through CMC were the individuals' frequency and duration of use with online communication. Other studies during the 1990's found similar results (e.g. Parks \& Roberts, 1998).

A comparison of the process of getting to know others through $\mathrm{CMC}$ versus $\mathrm{FtF}$ interactions was beginning to be researched during the early 2000's. Tidwell and Walther (2002) used an experimental design to examine the differences in disclosure and impression formation between FtF and CMC interactions with a "get acquainted" paradigm. Participants interacted in opposite-sex dyads with unacquainted others. Half of the participants were randomly assigned to interact via email accounts that were set up for the experiment, whereas the other half interacted FtF. The participants were instructed 
to get to know one another and work on a decision-making problem to encourage conversation and form a joint decision. FtF interaction partners were given 15 minutes to interact and $\mathrm{CMC}$ interaction partners were given 60 minutes (due to the time it takes to share similar amounts of information via text-only $\mathrm{CMC}$ compared to FtF). After the interaction, participants completed measures to assess perceptions of conversational effectiveness, conversational appropriateness, post-interaction uncertainty about participants' interaction partners, and own/partner self-disclosure. Tidwell and Walther (2002) also used content analysis with two trained judges to code for self-disclosures. The results of this study showed that participants within CMC dyads used a significantly greater proportion of questions and produced a significantly greater proportion of selfdisclosures compared to their FtF counterparts; which was determined through both selfreports and content analyses of the conversations (Tidwell \& Walther, 2002). The researchers reported that participants in CMC dyads used deeper probes and questions than participants interacting in FtF dyads. The researchers speculated that deeper probes may have been utilized by participants interacting via $\mathrm{CMC}$ to discover information about the other that is readily apparent during FtF interactions (e.g. physical appearance, age, etc.) but not apparent in text-based CMC. For example, the question of "are you male or female?" may be effective online, but would be an inappropriate question to ask offline. Although no differences were found between participants in $\mathrm{CMC}$ dyads versus $\mathrm{FtF}$ dyads in the levels of knowledge perceived to be gained about their interaction partners, but $\mathrm{CMC}$ respondents believed that the $\mathrm{CMC}$ environment hindered their ability to get to know their partner to a greater degree than did those interacting in the FtF condition. Tidwell and Walther (2002) concluded that participants interacting in CMC dyads 
appeared to overcome the limitations of visual and auditory anonymity and adapt to the medium in order to get to know one another. The limitations of CMC may have been overcome by asking more questions and using deeper probes.

After it had been established that relationships do in fact form online (e.g. Parks \& Floyd, 1996; Parks \& Roberts, 1998), researchers began to investigate the reasons why online relationships seem to form so easily. McKenna et al. (2002) speculated that individuals are motivated to make the relationships that they form online an important part of their identity and a social reality within their offline lives. To examine this issue, McKenna et al. (2002) conducted a longitudinal study of newsgroup members over two years. The results indicated that at Time 1 participants reported bringing online friends into their offline lives, that online relationships develop intimacy and closeness faster than their FtF counterparts, and that close and meaningful relationships do form online. Over half of the participants also reported meeting FtF with the individual they first met online. A two-year follow up on these newsgroup members revealed that $71 \%$ of the romantic relationships formed at Time 1 were still together and that $84 \%$ of participants reported their online relationships to be as real, important, and close as their offline relationships.

McKenna et al. (2002) also conducted an experiment to examine the differences in liking between participants who interacted with another online versus participants who interacted with another offline. Participants were placed in cross-sex dyads and instructed to "get acquainted with one another" over the course of two 20-minute interactions (McKenna et al., 2002). There were three conditions in this study. The first was the control condition which consisted of two FtF interactions. The second was the IRC 
(internet chat room) condition which had participants interact via CMC (text-based) first and then FtF for the second interaction, both with the same individual. Finally, participants in the "trading places" condition interacted with one individual FtF or CMC at the first interaction, switched communication mediums, and interacted with a "different" individual through FtF or CMC at the second interaction. Although the participants in this third condition believed that they were interacting with two different people in each interaction, they actually interacted with the same person in both interactions. The order of FtF and $\mathrm{CMC}$ interactions was counterbalanced to reduce the influence of order effects. The results of this study showed that participants in the IRC condition reported increased liking after meeting FtF (McKenna et al., 2002). Participants in the FtF condition showed a slight decrease in liking between the first and second interactions, but the participants in the IRC and FtF conditions did not differ in liking after the first interaction. After the second interaction, participants who originally met via $\mathrm{CMC}$ reported significantly more liking for their partners than those who met FtF in both interactions. The participants in the trading places condition reported increased liking for their interaction partner in later $\mathrm{FtF}$ interactions after communicating via $\mathrm{CMC}$ for the first interaction. The participants were unaware they were interacting with the same participant during each interaction, but reported liking the interaction partner significantly more after CMC interactions than after FtF interactions. Participants believed they knew their $\mathrm{CMC}$ partner more than they knew their FtF partner (same person) and also were more likely to tell their CMC partner specifically what they liked about them. This study suggests that there may be different aspects of $\mathrm{CMC}$ and FtF interactions that lead to similar levels of liking for participants' interaction partners. The 
finding that participants in both the FtF and IRC (CMC) conditions reported the same level of liking for their partners after the first interaction was investigated in the current research.

A similar study was conducted by Mallen et al. (2003), who used a text-based $\mathrm{CMC}$ condition along with a FtF condition where participants were given 30 minutes in order to "get to know one another" within each condition. Following the interaction, participants completed measures of satisfaction, closeness, recalled facts about the interaction partner, self-disclosure, conflict, and emotional understanding. The participants in the FtF condition reported greater satisfaction, levels of closeness, recall of facts immediately after the interaction, and self-disclosure in regard to the interaction. The participants who interacted FtF also reported less conflict than participants in the $\mathrm{CMC}$ condition. The researchers attributed the greater amount of conflict via $\mathrm{CMC}$ to the lack of contextual cues within the communication medium (Mallen et al., 2003). Ratings of self-disclosure and partner disclosure were highly correlated; partners agreed more on the level of disclosure (self and partner) via CMC compared to FtF. The two groups showed no difference in regard to accurately rating their partners' emotions, although FtF respondents reported more affect (both positive and negative) than $\mathrm{CMC}$ respondents.

The studies discussed above have employed only text-based CMC, but visual and auditory capabilities were becoming available during the early 2000's. Audio and visual capabilities allow for more interactivity with more cues being available (Ramirez \& Burgoon, 2004). Communication in the initial stages of a relationship influences how the relationship forms throughout the following interactions and may differ depending on the communication medium in which the initial interaction takes place (Ramirez \& Burgoon, 
2004). To examine differences between $\mathrm{CMC}$ and FtF in initial interactions, Ramirez and Burgoon (2004) had 80 same-sex dyads interact. Dyads were randomly assigned to either a FtF, text chat $\mathrm{CMC}$, audio $\mathrm{CMC}$, or video $\mathrm{CMC}$ condition and were instructed to "get acquainted with one another." The results revealed that the participants in the text chat CMC condition reported the least involvement (e.g. behavioral, cognitive, or emotional engagement; interdependence) within the interaction and had the greatest post-interaction uncertainty (e.g. lack of knowledge) about their interaction partners. The participants in the video CMC condition reported greater uncertainty about their interaction partners than the participants in the FtF condition. Visual cues reduced uncertainty more effectively than text-only, but did not reduce uncertainty as well as interacting FtF.

In sum, the early research on $\mathrm{CMC}$ has yielded mixed results, with some studies reporting greater liking for interaction partners via CMC (McKenna et al., 2002) and other studies reporting greater liking for interaction partners via FtF (Mallen et al., 2003). $\mathrm{CMC}$ has evolved from being a largely text-only environment to a communication medium that includes visual and auditory cues (Ramirez \& Burgoon, 2004). Many experimental studies, reviewed previously, have employed a "get acquainted" paradigm (Mallen et al., 2003; McKenna et al., 2002; Ramirez \& Burgoon, 2004) whereas some studies combined the "get acquainted" paradigm with a decision making problem (Tidwell \& Walther, 2002). The scholars who researched CMC throughout the late 1990's reported it as a cold and impersonal environment whereas research during the early 2000's suggested more positive conclusions (Sherman, 2003). Fifteen years ago, Parks and Floyd (1996) reported results that suggested that individuals' frequency and duration of internet use were the best predictors of whether they form relationships 
online. As the internet and $\mathrm{CMC}$ have become more integrated into society, more recent research may paint a different picture regarding CMC and the effects it has on social relationships. As CMC began to be viewed through a more holistic account that did not look at $\mathrm{FtF}$ and $\mathrm{CMC}$ interactions occurring in isolation but rather as complementary to one another in social relationships (Boase \& Wellman, 2005), researchers began to shed light on the social affordances (e.g. contact with family/friends not in physical proximity) of CMC that differ from FtF.

\section{Recent CMC Research}

A more recent approach to the study of CMC has taken into account that the internet has become a part of everyday life (Mesch \& Talmud, 2006). As more individuals are using $\mathrm{CMC}$ to form and maintain relationships (e.g. Boase \& Wellman, 2005), a different approach, that utilizes how CMC has been integrated into people's daily lives, has been taken in $\mathrm{CMC}$ research. The understanding that $\mathrm{CMC}$ has become more prevalent may help explain why $\mathrm{CMC}$ interactions are different from $\mathrm{FtF}$ interactions regarding self-disclosure and liking for interaction partners. The perceptions individuals have of $\mathrm{CMC}$ and $\mathrm{FtF}$ may influence the effects the medium of communication have on their interactions by possibly altering individuals' expectations of their interactions across these different communication mediums (Peter \& Valkenburg, 2006). Peter and Valkenburg (2006) speculated about characteristics of CMC that may result in different expectations of CMC interactions compared to FtF interactions. First, $\mathrm{CMC}$ has much more controllability than $\mathrm{FtF}$ in that participants can easily control whether, when, how, how much, and what they communicate. Second, CMC conveys fewer social status cues than FtF and may therefore result in more reciprocity. Third, 
individuals can more easily overcome shyness due to a lack of social presence in CMC. In regard to self-disclosure, individuals are able to talk about a greater number of topics (breadth) and also become less inhibited to disclose personal or intimate information (depth) within CMC interactions (Peter \& Valkenburg, 2006). Research on these speculations was soon to follow.

Researchers who employed the hyperpersonal perspective (Walther, 2007), which was emphasized in early research, stated that the dynamics of the sender, receiver, channel through which the sender and receiver interact, and feedback loop of expected response lead to exaggerated impressions and idealization through CMC. The hyperpersonal perspective was used to explain why CMC leads to closer relationships more quickly than FtF (Whitty, 2008). Strategic self-presentations via CMC, allowed by the extra time a user has to reflect on how to present oneself, were thought to create false or exaggerated impressions. Recent research (Back et al., 2010) has suggested that individuals display no evidence of self-idealization (exaggerated positive presentations) and present their personalities accurately and comparably in $\mathrm{CMC}$ and FtF encounters. Individuals are no longer as anonymous in today's world of CMC (Tufekei, 2010) compared to the CMC that was studied in early research which was largely anonymous and lacked many social consequences (Bargh, McKenna, \& Fitzsimons, 2002).

Recent research has also begun to focus on the complementarity of FtF and CMC interactions with the two modalities of communication no longer being distinct entities and individuals using them both to communicate with the same individual at different times. A majority of CMC interactions are with individuals known offline (Tufekei, 2010), and it has become very probable that FtF meetings complement $\mathrm{CMC}$ interactions 
once a social relationship has been established (Ramirez \& Zhang, 2007). Social presence theory (Ramirez \& Zhang, 2007; Whitty, 2008) states that the more social cues available in the interaction, the more social presence. Generally, it is thought that FtF involves greater social presence than $\mathrm{CMC}$ due to the lack of cues in $\mathrm{CMC}$. Information provided by verbal and nonverbal cues is of central influence when increasing partner predictability, social information exchange, self-disclosure, and attraction and may help explain why relationships formed through $\mathrm{CMC}$ are often taken to an offline context at some point (Whitty, 2008).

As mentioned above, audio/video capabilities were being introduced during this time. Ramirez and Zhang (2007) studied the effects of modality switching between FtF and $\mathrm{CMC}$ (text-only) on intimacy and social attraction. Modality switching refers to alternating between communication mediums. These researchers had multiple conditions involving two interactions that included FtF only in both interactions, FtF followed by $\mathrm{CMC}, \mathrm{CMC}$ followed by FtF, and CMC only in both interactions. The results showed that the participants who interacted via CMC for both interactions reported the highest intimacy and social attraction. Introducing FtF interactions to those that began in $\mathrm{CMC}$ hindered the further development of intimacy and social attraction between the interaction partners, and the participants reported no changes in intimacy or social attraction between the two interactions. Switching from FtF to CMC did not appear to alter the communication much either and resulted in no differences between the first and second interactions. These results suggest that the medium through which the initial interaction takes place, compared to extended interaction, has the greater impact regarding intimacy and social attraction on participants' ratings of their interaction 
partners because no differences were found between the first and second interactions when participants switched communication modalities.

Okdie et al. (2011) employed a similar paradigm of modality switching. Okdie et al. (2011) asked participants to get-acquainted with one another through two different orders of interaction sequence. The participants either interacted via CMC or FtF first, and then switched modality for the second interaction. The researchers counterbalanced the order to reduce primacy effects. The results showed that participants reported greater liking for their interaction partners and enjoyment of the interaction after interacting $\mathrm{FtF}$ than after interacting via CMC. FtF interactions were rated as more "difficult" than CMC interactions due to being more effortful to maintain the conversations, more difficult to come up with discussion topics, and more difficult to keep the conversation going. These results also shed light on why participants rated the FtF interactions more positively than the CMC interactions. Participants reported that their FtF partners were less self-centered and that they themselves felt less self-aware when interacting in the FtF condition.

In sum, the recent research on CMC has also yielded mixed results regarding intimacy and liking of interaction partners, with some researchers reporting positive results about CMC interactions (e.g. higher intimacy and social attraction; Ramirez \& Zhang, 2007) and others reporting positive results about $\mathrm{FtF}$ interactions (e.g. greater liking and enjoyment; Okdie et al., 2011). It remains unclear what the differentiating factor between these two modes of communication is in regard to the differences in liking of interaction partners found in previous studies. I argue that there are two different factors related to liking that are operating within $\mathrm{CMC}$ and $\mathrm{FtF}$ interactions; such that self-disclosure may lead to liking via CMC whereas responsiveness may lead to liking 
via FtF interactions. Self-disclosure and responsiveness are two related but distinct concepts that may operate differently in CMC and FtF interactions. Self-disclosure and responsiveness will be discussed in the following sections.

\section{Self-Disclosure}

Self-disclosure is an important part of social relationships and has been shown to lead to increased liking of one's partner. Collins and Miller (1994) conducted an extensive meta-analysis on the relationship between self-disclosure and liking. The authors defined self-disclosure as the revelation of personal information about oneself to another. Self-disclosure is viewed as a positive or rewarding outcome that communicates liking. Collins and Miller (1994) described three possible paths to understand the disclosure-liking link: we like others who disclose to us; we disclose to people we like; and we like people as a result of disclosing to them. These paths do not operate in isolation but are likely to be interconnected at many points in a relationship (Collins \& Miller, 1994).

Self-disclosure includes both descriptive and evaluative information (Collins \& Miller, 1994). Individuals tend to form positive impressions of those who self-disclose to them, and the self-disclosers are seen as more trusting, friendly, and warm (Collins \& Miller, 1994). Individuals also tend to self-disclose to others whom they already like and trust. Acquaintance paradigms employing previously unacquainted participants have also shown that liking leads to self-disclosure, that self-disclosure leads to liking, and that individuals like one another as a result of self-disclosing to them (Collins \& Miller, 1994). Several explanations have been provided as to why self-disclosure and liking are related to one another. It is possible that we self-disclose to communicate liking or that 
we self-disclose hoping it will be reciprocated. Self-disclosure is important in relationship formation, fosters intimacy, and has been observed to create interdependence among interaction partners (Shouten, Valkenburg, \& Peter, 2009).

What effect does the communication medium have on levels of self-disclosure? The level of self-disclosure has been examined comparing CMC and FtF interactions. Joinson (2001) examined self-disclosure comparing FtF and CMC interactions focusing on private self-awareness and visual anonymity as the variables leading to increased selfdisclosure via CMC. Joinson (2001) suggested that CMC results in high levels of selfdisclosure and that $\mathrm{CMC}$ users overestimate their input within these interactions compared to FtF interactions. Joinson (2001) conducted a series of studies to test these predictions. The first study used unacquainted participants who were placed in same-sex dyads in either a FtF condition or a text-only CMC condition. The participants were instructed to work on a nuclear war dilemma game to encourage communication and make a joint decision of which individuals they would choose to survive a nuclear holocaust. The researcher coded the conversations for the number of self-disclosures (Joinson, 2001). The results of the first experiment showed that participants in the FtF condition spoke more than the participants in the CMC condition, but participants in the $\mathrm{CMC}$ condition self-disclosed significantly more than participants in the $\mathrm{FtF}$ condition, which was measured with both content analyses of the interaction and self-report. In a second study, Joinson (2001) once again used unacquainted same-sex participants with the same instructions, but altered the communication medium. The $\mathrm{CMC}$ condition that had been used in the first study was divided into two CMC conditions for comparison. One $\mathrm{CMC}$ condition remained the text-only $\mathrm{CMC}$ that was 
used in the first study, but the comparison group in the second study was a CMC visually non-anonymous group with a video feed of each other (similar to Skype). The results showed that participants in the video CMC condition self-disclosed significantly less than participants in the text-only CMC condition. Joinson (2001) posed a few alternative explanations for these results. One is that people simply have more to self-disclose when anonymous. Another is that the video feed raised self-presentational concerns and resulted in higher public self-awareness.

A more recent investigation tested the differences in self-disclosure between textonly CMC, visual CMC, and FtF (Shouten et al., 2009). The participants in this study were paired in unacquainted cross-sex dyads and instructed to "get to know one another." The results showed that participants in both CMC conditions produced more selfdisclosure and direct questioning than participants in the FtF condition, measured with content analyses and self-report. The researchers speculated that direct questioning may mediate CMC's effect on self-disclosure (Shouten et al., 2009). The authors concluded that enhanced self-disclosure via text-only CMC cannot be attributed to control over selfpresentation and that reduced audio/visual cues are not what lead to enhanced selfdisclosure because there were no differences found between the two CMC conditions (text vs. video) regarding the level of self-disclosure.

Intimacy is related to increased levels of self-disclosure (Jiang, Bazarova, \& Hancock, 2010). Intimacy is defined as the closeness or connectedness within a social relationship (Sternberg, 1988). The relation of intimacy to the level of self-disclosure via CMC compared to FtF interactions was investigated by Jiang et al. (2010). Jiang and colleagues (2010) used a confederate to manipulate the intimacy of interaction partners 
by having the confederate exhibit different levels of self-disclosure. The researchers employed a text-based chat (CMC) condition and a FtF condition and manipulated whether the confederate was high or low in self-disclosure. Participants were told to complete a task and compile 10 tips for incoming freshman to survive college. When participants interacted with a low self-disclosure confederate, there were no differences found in intimacy between participants in the FtF and CMC conditions (Jiang et al., 2010). When participants interacted with a high self-disclosure confederate, participants rated the conversation as much more intimate via $\mathrm{CMC}$ compared to FtF. The results led the researchers to conclude that CMC intensifies the disclosure-intimacy link, especially at high levels of self-disclosure.

The research discussed above on the effects of CMC on self-disclosure has yielded mixed results. Some studies suggest that it is visual anonymity that leads to increased disclosure (e.g. Joinson, 2001), whereas others have found no differences in self-disclosure between text and video/audio CMC (e.g. Shouten et al., 2009). Selfconsciousness and self-awareness in relation to self-disclosure may help explain the differences in self-disclosure across alternate communication mediums cited above, which is discussed in a section to follow. The current research addressed this issue and investigated the factors influencing $\mathrm{CMC}$ interactions that differentiate these interactions from $\mathrm{FtF}$ interactions regarding self-disclosure.

Kim and Dindia (2011) conducted a meta-analysis of the literature regarding selfdisclosure and CMC. The literature reviewed included some studies that showed FtF interactions resulting in more self-disclosure, other studies showed no differences between FtF and $\mathrm{CMC}$ interactions, and still other studies showed $\mathrm{CMC}$ interactions 
leading to more self-disclosure. The authors concluded that CMC offers individuals the ability to disclose information about themselves, but studies have yielded mixed results concerning what is occurring via CMC that leads to differences in self-disclosure in comparison to FtF interactions. However, only 11 studies were included in the metaanalysis which leaves a large gap in the literature to be filled. One explanation for the inconsistent findings may be perceived partner responsiveness which will be discussed in the following section.

\section{Responsiveness}

Reis et al. (2004) defined perceived partner responsiveness as the "process by which individuals come to believe that partners both attend to and react supportively to central, core defining features of the self" (p. 203). Perceived partner responsiveness may also help explain the diverse findings reported throughout CMC literature. The effect of perceived partner responsiveness has not yet been investigated in a comparison of interpersonal outcomes between $\mathrm{CMC}$ and $\mathrm{FtF}$ communication. A discussion of the importance of responsiveness dates back to Mead's (1934) early work involving symbolic interaction. A central component of symbolic interaction is that we learn about ourselves through the responses provided by others. Responsiveness has consistently been found to be a key component in functioning relationships (Clark \& Lemay, 2010). Feeling understood by a partner contributes to the belief that the partner is motivated to be responsive, and that closeness can be defined in terms of responsiveness (Clark \& Lemay, 2010). Responsiveness includes the content of the response, the style of the response, the timing of the response, and is critical for understanding the self-disclosure process (Greene et al., 2006). 
Intimacy is also the result of an interpersonal process involving self-disclosure and partner responsiveness (Laurenceau, Barret, \& Pietromonaco, 1998). The Interpersonal Process Model of Intimacy sheds more light on the process of intimacy involving self-disclosure and perceived partner responsiveness. The model suggests that one individual will reveal personal information to another, and then the listener is expected to respond to the speaker by reciprocating the self-disclosure. The listeners are also expected to show that they understand, accept, and validate the individual's disclosure, and also feel positive towards the speaker (Laurenceau et al., 1998). The selfdiscloser's interpretations of the listener's response may be more important than the actual self-disclosure or the actual response. This model suggests that higher selfdisclosure and higher perceived partner responsiveness will lead to more intimacy. Selfdisclosure and responsiveness can operate over both $\mathrm{CMC}$ and $\mathrm{FtF}$ interactions, but it is not known whether they operate differently across the two communication mediums.

The norm of reciprocity applies to both self-disclosure and responsiveness (Clark \& Lemay, 2010), and responsiveness is important to whether self-disclosure is reciprocated (Laurenceau \& Kleinman, 2006). Partners feel the need to reciprocate responsiveness, but responsiveness generally is relationship-specific. Humans simply do not have the capabilities to be highly responsive to numerous people. The relationshipspecific aspect of responsiveness is important to consider when employing a "get acquainted" paradigm with two individuals who have never met before. The expectations of responsiveness are likely to be low in such relationships, but perceived responsiveness remains an important determinant of the formation of these relationships (Reis et al., 2004). 
Responsiveness communicates understanding, validation, and caring in response to a self-disclosure; and individuals depend on nonverbal cues to know how to appropriately respond (Laurenceau \& Kleinman, 2006). Responsiveness can be viewed in two different ways. Perceived responsiveness is how much one perceives the interaction partner as being responsive while actual responsiveness is how responsive the partner actually is during the interaction. Perceived responsiveness is generally measured with self-reports while actual responsiveness would be coded by independent raters as a content analysis. Misunderstanding nonverbal cues may lead to the differences between perceived and actual responsiveness, but perceived responsiveness may be more important to developing intimacy than actual responsiveness (Laurenceau \& Kleinman, 2006). An aspect of social interactions that is important to consider in the current research is that individuals' awareness of their interaction partners may influence perceived responsiveness (Reis et al., 2004). CMC leads to heightened levels of private self-awareness (Joinson, 2001; Yao \& Flanagin, 2006) and may lead individuals interacting via $\mathrm{CMC}$ to be more focused on themselves than to being focused on their interaction partners. A lower awareness of one's interaction partner may lead to lower levels of perceived partner responsiveness via CMC. It is possible that the lack of perceived partner responsiveness is why some research has found FtF interaction partners to report greater liking of one another (e.g. Okdie et al., 2011) in comparison with CMC interaction partners (i.e., there might have been lower levels of perceived responsiveness in the CMC condition). Self-consciousness and self-awareness may influence both selfdisclosure and perceived responsiveness, which will be discussed in the following section. 


\section{Self-Consciousness and Self-Awareness}

When in the presence of others, people can become self-conscious (Fenigstein, 1979). Self-consciousness can be divided into two separate components -- private and public. Private self-consciousness is characterized by covert aspects of the self (aspects not apparent to others; e.g. thoughts, feelings, emotions, etc.). Individuals high in this trait self-disclose more information than those low in this trait (Reno \& Kenny, 1992). Public self-consciousness is characterized by an individual's dispositional tendency to attend to the self as a social object. Individuals high in this trait are concerned about impressions, presentation, and over perceive themselves as the target of social evaluation (e.g. believing others are evaluating them). There are many reasons why high private selfconsciousness is related to higher levels of self-disclosure such as those higher in private self-consciousness have more self-knowledge and hence more to self-disclose. Other reasons given for why high private self-consciousness is related to higher levels of selfdisclosure include: self-information is immediately salient, self-disclosure fulfills the need to express oneself, and self-disclosure fulfills a need to understand oneself (see Davis \& Franzoi, 1987 for a review).

Self-consciousness and self-awareness are related constructs that share many similarities. Davis and Franzoi (1987) explain this by stating that private selfconsciousness is a "dispositional tendency to engage in a particular kind of psychological state termed private self-awareness" (p. 60). Private self-awareness is considered a state that occurs during interactions which is defined by attention to private aspects of the self; such as emotional states, motives, and reflections on past experiences. Displaying higher trait private self-consciousness leads individuals to become more state privately self- 
aware. The relationship between trait self-consciousness and state self-awareness may help to understand the effect that communication modality has on state self-awareness. CMC may lead to heightened levels of state private self-awareness, but these effects of CMC may be intensified by high dispositional trait private self-consciousness. Reno and Kenny (1992) suggested that public and private self-consciousness affect the initial acquaintance process differently. A study was conducted that used the Revised Self-Consciousness Scale (RSCS) to measure private and public selfconsciousness and social anxiety. Participants were unacquainted individuals who engaged in an initial interaction with one another. Participants' own private selfconsciousness was found to be positively correlated with self-reported self-disclosure. Furthermore, individuals high in social anxiety were less open and conveyed less information than individuals low in social anxiety. The authors concluded that the actual interaction may cause the self-conscious trait to influence participants more than a selfreport measure by having them focus on the interaction that just took place instead of retrospective self-report data based on past interactions. Retrospective self-reports may be influenced by different contextual factors than during an actual interaction, and therefore may be measuring different things. When an individual is not interacting with another, they may be experiencing low levels of state private self-awareness. The reason for the different experience of state self-awareness may result from the trait selfconsciousness and state self-awareness relationship mentioned above. The actual interaction may have influenced state self-awareness differently than a retrospective account of an individual's past state. 
Matheson and Zanna (1988) provided the first work involving a self-awareness approach to $\mathrm{CMC}$ and was based on the suggestion that $\mathrm{CMC}$ alters interpersonal communication because only written information was transmitted during the late 1980's; hence reducing the cues available via $\mathrm{CMC}$ compared to $\mathrm{FtF}$ communication and altering an individual's attention with less focus on others and more focus on oneself. In Matheson and Zanna's (1988) research, the public self was defined as overt aspects of the self that are sensitive to attention and evaluation by others such as self-presentation and social comparison. In contrast, the private self was viewed as a focus on internal needs and standards. Self-report measures of private and public self-awareness were given to participants after they communicated in either a text-only $\mathrm{CMC}$ or in a $\mathrm{FtF}$ condition. The results showed that participants in the $\mathrm{CMC}$ condition reported significantly higher private self-awareness and marginally lower public self-awareness than participants in the FtF condition (Matheson \& Zanna, 1988).

Self-awareness related to alternate communication mediums continued to be explored as these forms of communication evolved and became more commonly used. Joinson (2001) measured self-awareness related to self-disclosure. In this study the researcher manipulated private and public self-awareness. Private self-awareness was manipulated by having the participants' own video on the screen (high) or by playing an episode of the Simpsons in the background (low). Public self-awareness was manipulated by having participants arrive in a darkened corridor with anonymity stressed by stating that they would never meet their interaction partner (low) or by having the participants arrive in a well-lit corridor with cameras present and researchers stating that they would meet their partner after the interaction (high). The communication medium used was text- 
only CMC. The results revealed that participants in the high private and low public selfawareness condition self-disclosed significantly more than participants in the high private and high public or low private and low public self-awareness conditions (Joinson, 2001).

Based on Joinson's (2001) study, Yao and Flanagin (2006) employed a similar self-awareness manipulation. Public self-awareness was manipulated by having a video of the participants on their own screen and telling them an audience was watching them. Private self-awareness was manipulated by having their own image on the screen and being told nobody else could see it. These researchers had participants work on a "desert survival problem" to make a joint decision on the 12 most important items to survival when stranded in the desert and then rate one another on dimensions of social attractiveness, intimacy, and politeness. The results of their study showed that high private and low public self-awareness led to the most social attraction, whereas low private and low public self-awareness led to the least social attraction. Individuals in the high public self-awareness condition were rated as less polite than those in the other conditions.

The studies examining the effect of self-awareness on self-disclosure reviewed above have yielded consistent results. High private self-consciousness as a trait and high private self-awareness as a state have consistently resulted in more self-disclosure than public self-consciousness and public self-awareness, respectively (Davis \& Franzoi, 1987; Joinson, 2001). The self-awareness manipulations used in previous research (e.g. mirror, camera, or tape recorder) have been successful in inducing both public and private state self-awareness (Yao \& Flanagin, 2006). The current research did not employ a manipulation of self-awareness but examined the effect that the communication 
medium had on state self-awareness. The lack of a self-awareness manipulation allowed for comparisons between natural $\mathrm{CMC}$ and $\mathrm{FtF}$ interactions to be drawn; similar to those encountered on a daily basis.

To summarize the literature reviewed in the preceding sections, early CMC research during the 1990's and early 2000's reported inconsistent results surrounding the effects that CMC has on social relationships. Researchers have recently begun to acknowledge that $\mathrm{CMC}$ interactions are often incorporated into the offline lives of internet users and individuals use $\mathrm{CMC}$ and FtF interactions complementarily (Ramirez \& Zhang, 2007). While the research regarding CMC's effects on self-disclosure has yielded mixed results (Kim \& Dindia, 2011), it becomes important to understand why the results thus far have been inconclusive. Perceived partner responsiveness has not been examined within the context of CMC and may have an effect on whether participants like their interaction partners more via CMC (McKenna et al., 2002; Ramirez \& Zhang, 2007) or more via FtF (Mallen et al., 2003; Okdie et al., 2011). In addition, trait selfconsciousness may be related to state self-awareness differently through alternate forms of communication by altering an individual's attention and therefore influencing the perceptions of one's own self-disclosure, partner self-disclosure, and perceived partner responsiveness which are related to the liking of an interaction partner. The current research addressed these issues.

\section{The Current Research}

The current study employed a "get acquainted" paradigm in which unacquainted participants interacted naturally within dyads randomly assigned to different communication mediums. Participants interacted in one of three conditions, differentiated 
by only the communication medium. The communication mediums were text-only CMC, video/audio $\mathrm{CMC}$, and FtF. Trait self-consciousness was measured prior to the interaction and perceived own self-disclosure, perceived partner self-disclosure, perceived partner responsiveness, and state self-awareness were measured following the interaction. Perceived own self-disclosure, perceived partner self-disclosure, perceived partner responsiveness, and state self-awareness were expected to vary across the different communication mediums. This prediction will be explained below.

As noted above, previous research comparing FtF interactions with CMC interactions have found mixed results concerning the effect of the communication medium on the level of self-disclosure (Kim \& Dindia, 2011). The methodologies that were used in these past studies may have led to conflicting results. Some studies used both self-report and content analysis (Tidwell \& Walther, 2002) whereas others used either self-report (Mallen et al., 2003) or content analysis (Joinson, 2001) alone. The current study employed a self-report measure of perceived own self-disclosure and perceived partner self-disclosure. Studies using self-report measures have found that CMC leads to higher levels of self-disclosure than FtF (Shouten et al., 2009; Tidwell \& Walther, 2002). Self-reports of own self-disclosure and perceived partner self-disclosure were expected to be greater via text-only $\mathrm{CMC}$ than video/audio $\mathrm{CMC}$ or FtF due to the lack of cues, resulting in participants having more to self-disclose. Therefore it was hypothesized that:

$H_{y p}{ }_{1}$ Participants within text-only CMC dyads will report the greatest levels of own self-disclosure and partner self-disclosure whereas participants within FtF dyads will report the lowest levels of own and partner self-disclosure. 
Participants within audio/video CMC dyads will report intermediate levels of own and partner self-disclosure.

However, whereas $\mathrm{CMC}$ was expected to lead to higher levels of perceived selfdisclosure than FtF, perceived partner responsiveness was expected to be reported at higher levels after interacting FtF compared to CMC. Social presence theory (Ramirez \& Zhang, 2007; Whitty, 2008) suggests that FtF interactions have greater levels of social presence than $\mathrm{CMC}$ interactions due to the lack of nonverbal and contextual cues available within CMC interactions. Laurenceau and Kleinman (2006) reported that responsiveness communicates understanding, validation, and caring in response to a disclosure, but more importantly, that individuals depend on nonverbal cues to know how to appropriately respond. The text-only $\mathrm{CMC}$ condition lacked auditory, visual, and nonverbal cues compared to the video/audio CMC and FtF conditions. Without these cues, individuals are less aware of their partners, which influences perceived partner responsiveness by not having nonverbal cues that are vital in order to be responsive (Reis et al., 2004). Therefore I hypothesized that:

$\mathrm{Hyp}_{2}$ Participants within FtF dyads will perceive the greatest levels of partner responsiveness whereas participants within text-only dyads will perceive the lowest levels of partner responsiveness. Participants within audio/video CMC dyads will report intermediate levels of partner responsiveness.

Differences in regard to state private self-awareness have been found across communication mediums. Previous research has found that $\mathrm{CMC}$ interactions result in higher levels of state private self-awareness than FtF interactions (Joinson, 2001; Matheson \& Zanna, 1988; Yao \& Flanagin, 2006). Due to the lack of cues involved in 
text-only $\mathrm{CMC}$, it was expected that participants would have more attention focused on themselves. When auditory and visual information becomes available, it is probable that participants would have more attention focused on their interaction partners and own selfpresentational concerns. Therefore, I hypothesized that:

$\mathrm{Hyp}_{3}$ Participants within text-only CMC dyads will report higher levels of state private self-awareness than participants within audio/video CMC dyads and participants within FtF dyads.

Perceived own self-disclosure, perceived partner self-disclosure, and perceived partner responsiveness were expected to relate to liking of participants' interaction partners. Studies have shown inconsistent effects of the communication medium on liking for one's interaction partner. Support for both CMC interactions related to increased liking compared to FTF interactions (e.g. McKenna et al., 2002; Ramirez \& Zhang, 2007) and $\mathrm{FtF}$ interactions compared to $\mathrm{CMC}$ interactions related to increased liking (e.g. Mallen et al., 2003; Okdie et al., 2011) have been found. The current research addressed differences between the communication mediums' effects on liking by examining selfdisclosure and perceived partner responsiveness. As noted earlier, self-disclosure is related to liking for a partner (Collins \& Miller, 1994). In addition, research has shown that perceived partner responsiveness is related to higher levels of intimacy and closeness (Laurenceau et al., 1998) and is an important component in the formation of relationships (Reis et al., 2004). It may be that self-disclosure and responsiveness both lead to liking, but influence the interactions to different extents through text-only CMC, visual/audio $\mathrm{CMC}$, and $\mathrm{FtF}$. Therefore the following research questions were posed: 
$R Q_{1}$ Are perceived own self-disclosure, perceived partner self-disclosure, and perceived partner responsiveness positively associated with liking for the interaction partner?

$R Q_{2}$ Does perceived own self-disclosure and perceived partner self-disclosure associate positively with liking to a greater extent for participants who communicate via text-only CMC than for participants who communicate FtF? $R Q_{3}$ Does perceived partner responsiveness associate positively with liking to a greater extent when participants communicate FtF than for participants who communicate text-only CMC?

Due to the inconsistent findings regarding liking for one's interaction partner across alternate communication mediums, I pose the following research question: $R Q 4$ Which communication medium (FtF, audio/video $C M C$, text-only $C M C)$ results in the most liking for the interaction partner?

An issue that has not been addressed thus far is the specific topics discussed during disclosure. Therefore, participants were asked how much they felt they knew about their interaction partner's hobbies/interests, family, major/job, and social life after the interaction. Due to no prior research being available on the topics of disclosure via $\mathrm{CMC}$ compared to $\mathrm{FtF}$ interactions, I posed the following research question: RQ5 Are there differences between FtF, audio/video CMC, and text-only CMC regarding the specific topics of self-disclosure?

Research has yielded inconsistent findings comparing FtF and CMC regarding self-disclosure (Kim \& Dindia, 2011), and responsiveness has yet to be compared across communication mediums. These inconsistencies may result from individuals perceiving 
the communication mediums differently and the effect the medium has on their ability to self-disclose and be responsive. Therefore, I pose the following research question: RQ6 Do individuals perceive text-only CMC as being a more difficult medium in which to self-disclose and be responsive to one's interaction partner compared to audio/video CMC and FtF? 


\section{CHAPTER III}

\section{METHOD}

\section{Participants}

Participants were recruited through the Psychology participant pool at a medium sized Midwestern university. Participants received course participation or extra credit for their participation in the research. A total of 146 individuals participated in the current study. Four individuals were deleted from the analyses due to having been acquaintances/friends prior to the experiment (a question in a post-interaction survey asked if they knew each other). One hundred and forty two individuals were included in the analyses yielding 71 unacquainted dyads. Participants ranged in age from 18 to 42 ( $M$ $=20.15, S D=2.53)$. Twenty four males $(16.9 \%)$ and 118 females $(83.1 \%)$ were paired in dyads consisting of 48 female-female dyads, 22 male-female dyads, and one male-male dyad. The sample's cultural diversity consisted of 113 White $(81.9 \%), 13$ Black $(9.4 \%)$, nine Hispanic/Latino (6.5\%), and three Asian individuals (2.2\%). Fifty one participants were Freshman (35.9\%), 29 Sophomore (20.4\%), 48 Junior (33.8\%), and 14 Senior (9.9\%). Participants were asked if they had ever used Skype or another web-based communication to interact with another. Twelve participants reported they had never used these communication mediums to interact with another individual $(8.5 \%), 29$ reported 
once or twice $(20.4 \%), 52$ reported a few times $(36.6 \%)$, and 49 reported several times $(34.5 \%)$.

\section{Materials}

The materials involved two computers, two web cams, Skype, a timer, and two separate but similar rooms for the CMC conditions. The participants in the video/audio CMC condition used Skype to communicate with video and audio capabilities and the participants in the text-only CMC condition also used Skype, but without audio/video capabilities.

\section{Procedure}

Participants arrived at two separate locations in the Psychology lab, were greeted by the experimenters, and led to separate rooms to complete a pre-interaction questionnaire. At this time, the experimenters read an overview of the study to the participants and had them sign an informed consent if they agreed to participate. Once consent was given to participate in the study, the participants were instructed to complete a pre-interaction questionnaire. After the pre-interaction questionnaire has been completed, the participants were notified of which condition to which they were randomly assigned. The conditions were identical regarding the instructions and only differed in the communication medium through which the participants interacted (textonly $\mathrm{CMC}$, audio/video $\mathrm{CMC}$, or FtF).

Once participants were assigned to a condition, the experimenter led them into the same room (FtF condition) or had them remain in separate rooms (CMC conditions) and instructed them to "get acquainted" through an unstructured natural interaction in which they were not restricted to a particular topic of discussion. The participants were given 20 
minutes to get acquainted with one another before the experimenter reentered the room to end the interaction. At this time, Skype was closed (CMC conditions) or the participants were led to separate rooms ( $\mathrm{FtF}$ condition) and instructed to complete a post-interaction questionnaire. After the post-interaction questionnaire, participants were debriefed and thanked for their participation.

\section{Pre-Interaction Measures}

Demographics. Participants were asked how familiar they are with CMC and their frequency of use. Age, sex, race/ethnicity, and year in school were also assessed.

Self-Consciousness and Social Anxiety. The Self-Consciousness Scale Revised (SCSR; Scheir \& Carver, 1985) was used to assess trait private and public selfconsciousness along with social anxiety. Examples of items on the scale are "I'm always trying to figure myself out" (Private Self-Consciousness -8 items $\alpha=.72$ ), "I care a lot about how I present myself to others" (Public Self-Consciousness -7 items $\alpha=.75$ ), and "It takes me time to get over my shyness in new situations" (Social Anxiety -6 items $\alpha=$ .70). The SCSR involves a four-point scale including "not like me at all", "a little like me", "somewhat like me", and "a lot like me."

\section{Post-Interaction Measures}

Self-Awareness. Items to address state private and public self-awareness during the interaction were modified from Yao and Flanagin (2006). The only modifications to the items were that "task partner" was replaced with "interaction partner" and "during the study" was replaced with "during the interaction" due to the nature of the current study. Examples of the items in this scale are "I found myself attending to my inner feelings" (Private Self-Awareness -5 items $\alpha=.72$ ) and "I felt that someone was watching me 
during the study" (Public Self-Awareness -5 items $\alpha=.65$ ). Items were responded to on a five-point scale ranging from "not at all" to "very much".

Intimacy and Social Attraction. Intimacy and Social Attraction were measured using scales developed by Yao and Flanagin (2006) with the same modifications mentioned in the self-awareness measures. This scale included items such as "my interaction partner was willing to self-disclose personal thoughts to me" (Intimacy - 8 items $\alpha=.71$ ) and "I think my interaction partner could be a friend of mine" (Social Attraction -5 items $\alpha=.72$ ). Items were responded to on a five-point scale ranging from "not at all" to "very much."

Perceived Self-Disclosure. Items for self-disclosure were borrowed from a previous study (Sprecher, Treger, \& Wondra, in press). Items included questions such as “How much did you tell your partner about yourself?" (Perceived Own Self-Disclosure 3 items $\alpha=.64$ ) and "How much did your partner tell you about himself or herself?" (Perceived Partner Self-Disclosure -3 items $\alpha=.71$ ). Perceived own self-disclosure and partner self-disclosure were rated on a seven-point scale ranging from "not at all" to "a great deal."

Perceived Partner Responsiveness. Perceived partner responsiveness was measured with a scale developed by Reis, Maniaci, Caprariello, and Eastwick (2011) for perceived responsiveness regarding initial interactions (12 items $\alpha=.94)$. Participants read “Compared to most experiences I've had meeting somebody new, I get the feeling that this person..." and responded to statements addressing perceived partner responsiveness. Example items from this scale include “...knows me well” and “...really 
listens to me." Statements were rated on a seven-point scale ranging from "not at all true" to "completely true."

Liking. Liking was assessed with four items (Sprecher et al., in press). Three items were rated on a seven-point scale ranging from "not at all" to "a great deal." An example of these items was "How much did you like the other?" Three items were rated on a seven-point scale ranging from "not at all" to "a great deal." A final item asked participants which statement best described their feelings about the other with statements ranging from 1-“I feel that I would probably dislike this person very much" to 7-“I feel that I would probably like this person very much.” The $\alpha$ was .87 for these four items.

Topics of Disclosure. Topics of disclosure were assessed with four items (Reis, et al., 2011). The topics included hobbies/interests, family, major/job, and social life. An example item was "How much do you think you know about the other's family?" These items were addressed on a seven-point scale ranging from "not at all" to "a great deal." Difficulty of Communication. Participants were asked if the medium through which they communicated made it easier or more difficult compared to the other communication mediums ( $\mathrm{FtF}$, audio/video $\mathrm{CMC}$, and text-only $\mathrm{CMC}$ ) to be responsive or to self-disclose. These two items were phrased as "Do you think the way you were asked to communicate made it easier or more difficult to be responsive?" The items were assessed on a five-point scale ranging from "easier" to "more difficult."

The means of all measures were calculated and used for analysis. 


\title{
CHAPTER IV
}

\section{RESULTS}

\begin{abstract}
Results
Results were analyzed using individual-level analyses, even though participants were paired within dyads. The current study assessed differences between individuals across communication mediums to detect differences between the communication medium's effect on interpersonal outcomes. Dyadic data analyses would have addressed alternate, more complex hypotheses and research questions which will be assessed in future research.
\end{abstract}

\section{Descriptive Statistics}

Table 1 reveals the descriptive statistics and correlations among the dependent measures of perceived self-disclosure, perceived partner self-disclosure, perceived responsiveness, liking, and private self-awareness used in the following analyses. An examination of the means revealed that participants reported generally high levels of liking for their interaction partner. Participants reported similar levels of own selfdisclosure and partner self-disclosure ( $M=4.90$ for both measures). Participants reported moderate degrees of responsiveness and private self-awareness on each of the respective scales. Self-disclosure, perceived partner self-disclosure, responsiveness, liking, and 
private self-awareness were all positively correlated with one another. Perceived selfdisclosure, perceived partner self-disclosure, responsiveness, and liking were significantly positively correlated at $p<.001$. Private self-awareness was positively correlated with self-disclosure $(p<.01)$, partner self-disclosure $(p<.05)$, and responsiveness $(p<.05)$, but was not correlated with liking.

\section{Tests of Hypotheses}

Table 2 reveals the means, standard deviations, and effect sizes of perceived selfdisclosure, perceived partner self-disclosure, perceived responsiveness, and private selfawareness across communication mediums. It was hypothesized that participants interacting via text-only $\mathrm{CMC}$ would report the highest levels of self-disclosure, partner self-disclosure, and private self-awareness; whereas participants interacting FtF would report the highest levels of responsiveness (Hyp 1-3). The medium through which participants interacted (text-only $\mathrm{CMC}$, audio/video $\mathrm{CMC}$, or FtF) was utilized as the independent variable for comparison. A One-Way Multivariate Analysis of Variance revealed significant differences across the three conditions, Wilks' $\Lambda=.76, F(8,272)=$ 5.03, $p<.001$. Perceived self-disclosure (partial $\eta^{2}=.18$ ) accounted for the strongest differences followed by partner self-disclosure (partial $\eta^{2}=.16$ ), responsiveness (partial $\left.\eta^{2}=.10\right)$, and private self-awareness (partial $\eta^{2}=.04$ ).

Bonferroni post-hoc analyses were conducted to analyze the differences across conditions for each dependent measure. In regard to perceived self-disclosure, participants in the FtF condition and audio/video condition did not differ, but both the participants in the FtF condition $(p<.001)$ and the participants in the audio/video condition $(p<.001)$ reported significantly higher levels than participants in the text-only 
condition. Perceived partner self-disclosure revealed a similar trend, with participants interacting FtF or participants interacting audio/video not differing from one another, but participants in the $\mathrm{FtF}$ condition $(p<.001)$ and the audio/video condition $(p<.001)$ reporting significantly higher levels of partner self-disclosure than participants in the text-only condition. Responsiveness also revealed no differences between participants in the FtF condition and participants in the audio/video condition. However, participants in both the $\mathrm{FtF}$ condition $(p=.001)$ and the audio/video condition $(p=.01)$ reported significantly higher levels of partner responsiveness than participants in the text-only condition, and perceived responsiveness was the only variable hypothesized resulting in the predicted direction. No significant differences were found between conditions in state private self-awareness, however. The hypothesis that FtF interactions would lead to greater levels of perceived partner responsiveness was supported. The hypotheses that text-only CMC would lead to the greatest levels of perceived self-disclosure and private self-awareness were not supported. The results suggest the FtF and audio/video interactions led to the greatest levels of perceived self-disclosure, whereas no significant differences regarding private self-awareness were found.

\section{Tests of Research Questions}

A multiple regression analysis was conducted to test the question of whether selfdisclosure, partner self-disclosure, and partner responsiveness are positively associated with liking for the interaction partner $(R Q 1)$. Reported levels of own self-disclosure, partner self-disclosure, and responsiveness significantly positively predicted liking of one's interaction partner, $R^{2}=.49, F(3,138)=44.51, p<.001$. Own self-disclosure was the only predictor that did not predict liking to a significant extent. Standardized $\beta$ s and 
significance tests can be found in Table 3. Separate multiple regressions were conducted with participants in each condition to examine differences between communication mediums on the predictive effect of perceived self-disclosure, perceived partner disclosure, and perceived responsiveness $(R Q 2 \& R Q 3)$. Within each condition, these three predictors remain statistically significant predictors of liking for one's interaction partner $(p s<.001)$. Table 4 reveals the inferential tests for each condition. The conditions vary in the degree to which perceived self-disclosure, perceived partner self-disclosure, and perceived responsiveness predict liking of one's interaction partner (differences in liking discussed below). Participants' reported responsiveness within the FtF and audio/video CMC conditions and partner self-disclosure in the text-only condition had the strongest positive relationships with liking. Perceived self-disclosure was negatively related to liking within the text-only CMC condition $(\beta=-.15)$ and audio/video CMC condition $(\beta=-.04)$, but was positively related within the $\mathrm{FtF}$ condition.

\section{Liking}

Differences in liking are addressed with the research question (RQ4) that there may be differences in liking across different communication mediums. A One-way Analysis of Variance was conducted to test these differences. Participants in the FtF condition $(M=5.09, S D=.93)$ reported more liking of their interaction partner than participants in the audio/video condition $(M=4.71, S D=1.03)$ or participants in the textonly condition $(M=4.32, S D=1.08), F(2,139)=7.01, p=.001$. Bonferroni post-hoc analyses revealed the difference between participants' reported liking within the FtF and text-only conditions was the only difference that reached significance $(p=.001)$. 


\section{Topics of Disclosure}

A Multivariate Analysis of Variance was conducted to test the question of whether individuals disclose more or less about certain topics across communication mediums. Means and standard deviations of hobbies/interests, family, major/job, and social life can be found in Table 5. Significant differences were found in topics of disclosure between communication mediums, Wilks' $\Lambda=.72, F(8,272)=6.20, p<.001$. How much participants felt they knew about their interaction partner's family (partial $\eta^{2}$ $=.17$ ) and social life (partial $\eta^{2}=.17$ ) accounted for a majority of the differences followed by major or job (partial $\eta^{2}=.04$ ) and hobbies or interests (partial $\eta^{2}=.02$ ). Bonferroni post-hoc analyses revealed significant differences between participants within the FtF condition and participants in the audio/video condition when compared to participants in the text-only condition in regard to family disclosure and social life disclosure with participants interacting $\mathrm{FtF}$ and audio/video resulting in higher levels of disclosure on these topics than text-only $(p s \leq .001)$. No significant differences between conditions were found regarding hobbies or interests disclosure and major or job disclosure $(p s>.10)$

\section{Difficulty of Communication Medium}

The differences across mediums in regard to perceived disclosure and responsiveness, discussed thus far, suggest that participants may have different perceptions of their ability to self-disclose and be responsive dependent on the communication medium. Participants were unaware of the other conditions when asked these questions to remain unbiased due to a lack of comparisons. A One-Way Multivariate Analysis of Variance was conducted with communication medium entered 
as the independent variable and difficulty of disclosure and responsiveness entered as dependent variables. Table 6 reveals the means and standard deviations of the dependent measures. Significant differences emerged between communication mediums in regard to reported difficulty of self-disclosure and responsiveness due to the communication medium, Wilks' $\Lambda=.90, F(4,276)=3.54, p=.008$. Difficulty of self-disclosure (partial $\eta^{2}=.07$ ) and responsiveness (partial $\eta^{2}=.08$ ) both contributed nearly equivalent amounts to this difference. Bonferroni post-hoc analyses of these differences revealed significant differences in participants' reported difficulty of self-disclosure $(p=.006)$ and responsiveness $(p=.002)$ between participants in the $\mathrm{FtF}$ condition and audio/video condition with audio/video being rated as more difficult. Participants in the text-only condition did not differ significantly from participants in the FtF or audio/video conditions. 


\section{CHAPTER V \\ DISCUSSION}

\section{General Discussion}

The purpose of the current experiment was to examine differences between communication mediums in perceived self-disclosure, perceived partner self-disclosure, perceived partner responsiveness, and state private self-awareness to reveal what differentiates alternate communication mediums from one another. Along with these variables, liking of one's interaction partner and perceived topics of disclosure were also examined to further investigate these differences. By examining these variables across different communication mediums, I was able to further understand the literature regarding comparisons of $\mathrm{CMC}$ and $\mathrm{FtF}$ interactions examining self-disclosure that have revealed inconsistent findings (Kim \& Dindia, 2011; Nguyen, Bin, \& Campbell, 2011). The inconsistent findings are likely the result of different methodological and theoretical approaches taken when conducting $\mathrm{CMC}$ and $\mathrm{FtF}$ comparisons. Therefore, I find it important to place the current discussion in the context of the methodology utilized in the current research. 


\section{Methodological Issues}

The current research employed a get-acquainted paradigm in which pairs of unacquainted individuals were instructed to interact with one another for 20 minutes to get to know one another. Participants were not given any instructions as to what they were to discuss and the interaction was completely unstructured as many initial interactions are outside of the laboratory. Following the 20 minute interaction, participants reported perceived self-disclosure, perceived partner self-disclosure, perceived partner responsiveness, state private self-awareness, liking, and perceived topics of disclosure. Measurement of disclosure and responsiveness were solely based on participants' perceptions of the interaction and no actual disclosure was recorded or analyzed. The difference between perceived (self-report) disclosure and responsiveness and actual (observational) disclosure and responsiveness becomes an important consideration when comparing $\mathrm{CMC}$ and $\mathrm{FtF}$ interactions due to perceived and actual measures being distinctively different from one another (discussed below). I believe these differences in measurement may be a prime culprit for what is leading to the inconsistent findings throughout the literature and advise the current discussion to be interpreted under the methodology described above.

\section{Hypotheses}

It was hypothesized that participants interacting via text-only $\mathrm{CMC}$ would report the highest levels of perceived self-disclosure, perceived partner self-disclosure, and state private self-awareness compared to participants interacting via audio/video CMC and FtF. It was also hypothesized that participants interacting FtF would report the greatest levels of perceived partner responsiveness. The hypotheses regarding perceived 
disclosure and state private self-awareness were not supported. The hypothesis that participants interacting $\mathrm{FtF}$ would report the highest levels of perceived partner responsiveness was supported.

Participants interacting via audio/video CMC reported the highest levels of perceived self-disclosure, but did not report significantly different levels of selfdisclosure compared to participants interacting FtF. The differences in perceived selfdisclosure emerged between participants interacting via audio/video $\mathrm{CMC}$ and $\mathrm{FtF}$ compared with participants interacting via text-only CMC. These results are in direct contrast to the hypotheses.

There are numerous reasons why these differences may have occurred. Differences between measuring perceived self-disclosure and not actual self-disclosure needs to be considered when interpreting this finding due to the two measures possibly measuring different constructs. When interacting via text-only CMC; age, sex, and location (ASL) are appropriate disclosure topics (Nguyen et al., 2011). ASL would likely be coded as self-disclosure if measuring actual self-disclosure via content analysis or alternate observational measures. One possibility is that participants did not perceive disclosing ASL as self-disclosure in my study. There are also theoretical implications that will be discussed below.

Participants interacting FtF reported the highest levels of perceived partner selfdisclosure, which did not support the hypothesis that text-only CMC would result in the highest levels of self-disclosure. Following a similar trend to perceived self-disclosure, there were not significant differences between participants interacting $\mathrm{FtF}$ and audio/video CMC in partner self-disclosure. The significant differences in partner self- 
disclosure emerged between participants interacting via FtF and audio/video CMC when compared to participants interacting via text-only CMC. The finding that participants perceived the highest levels of partner self-disclosure when interacting FtF is likely the result of similar reasoning as the findings regarding perceived self-disclosure, such as perceived versus actual self-disclosure measures resulting in different constructs being measured.

The hypothesis that participants interacting FtF would report the highest levels of perceived partner responsiveness was supported. FtF interactions involve many more nonverbal and contextual cues compared to text-only CMC, whereas audio/video CMC interactions appear to be more similar to FtF interactions than text-only $\mathrm{CMC}$ interactions. Following a similar trend as perceived own and partner self-disclosure, the significant differences in responsiveness were found between participants interacting FtF and via audio/video CMC when compared to participants interacting via text-only CMC. Social Presence Theory (Whitty, 2008) states that interacting FtF involves more social presence than interacting via audio/video $\mathrm{CMC}$, which involves more social presence than interacting via text-only CMC. The lack of nonverbal and contextual cues when interacting via text-only $\mathrm{CMC}$ is likely the reason for the lack of perceived responsiveness when interacting through this medium. The inability to be responsive through text-only CMC also is likely to hinder the perceptions of partner responsiveness. Individuals are unable to employ nonverbal communication through text-only $\mathrm{CMC}$, and therefore must rely solely on text to communicate responsiveness. 


\section{Research Questions}

The research questions addressed in the current research involved perceived selfdisclosure, perceived partner disclosure, and perceived partner responsiveness as positive predictors of liking for one's interaction partner. A regression analysis of these three predictors on liking revealed perceived partner self-disclosure and perceived responsiveness as significant positive predictors of liking, whereas perceived selfdisclosure was not a significant predictor of liking. Perceived responsiveness was the strongest predictor of liking for one's interaction partner. Implications of this finding for close relationship and $\mathrm{CMC}$ research is provided below.

Separate regression analyses were conducted to test differences in predicting liking across $\mathrm{FtF}$, audio/video $\mathrm{CMC}$, and text-only $\mathrm{CMC}$ interactions. Responsiveness was the only significant predictor of liking within the FtF and audio/video CMC conditions, whereas both partner self-disclosure and responsiveness were significant predictors of liking in text-only CMC interactions. Perceived level of one's own selfdisclosure was not a significant predictor of liking in any condition. Within each condition, these three predictors were positively associated with liking of one's interaction partner with the exception of perceived self-disclosure reported by participants interacting via text-only $\mathrm{CMC}$ or audio/video CMC, which had a negative relationship with liking (although not significant). The negative relationship between perceived self-disclosure and liking may have occurred for the same reason as the differences in levels of perceived disclosure discussed above. Participants may not enjoy disclosing ASL, and therefore may lead to decreased levels of liking for one's interaction partner. The finding that responsiveness was the only consistent significant positive 
predictor of liking across all three conditions sheds light on a novel approach to comparing $\mathrm{CMC}$ and $\mathrm{FtF}$ interactions due to the lack of including this variable in $\mathrm{CMC}$ research. FtF interactions allow for more responsiveness than audio/video CMC, which allows for more responsiveness than text-only CMC. The results of the current study support this reasoning with the strongest positive relationship between responsiveness and liking via FtF interactions, followed by audio/video $\mathrm{CMC}$ interactions, followed by text-only CMC interactions.

Participants interacting FtF reported the highest levels of liking for their interaction partner. This finding supports Mallen et al.'s (2003) and Okdie et al.'s (2011) conclusion that FtF interactions lead to increased liking for one's interaction partner. The reasons for this are likely the higher levels of perceived self-disclosure and responsiveness reported after interacting FtF. Participants also rated FtF interactions as being easier than text-only $\mathrm{CMC}$ and audio/video $\mathrm{CMC}$ and the ease of disclosing information and being responsive may have led to heightened levels of liking for one's interaction partner.

An issue that has not been addressed in the literature thus far is the specific topics of disclosure in research comparing FtF and $\mathrm{CMC}$ interactions. Participants in all conditions disclosed the most information about their major or job. Participants interacting FtF disclosed more information about their families and social life compared to other topics, which led to the largest differences between communication mediums regarding topics of disclosure. 


\section{Implications}

The current research addressed hypotheses and questions that have been asked in prior research. Due to the importance of methodological and theoretical issues involved in conducting research, they are addressed in the following section after a review of the implications for CMC research is discussed.

\section{Communication Medium}

The current research has numerous implications for research concerning the comparison of $\mathrm{FtF}$ and $\mathrm{CMC}$ interactions. Early $\mathrm{CMC}$ research suggested that $\mathrm{CMC}$ offers the ability to disclose information (McKenna et al., 2002), but failed to account for the medium's hindering effect on the ability for interaction partners to be responsive to one another. Recent literature (Clark \& Lemay, 2010; Laurenceau et al., 1998;

Laurenceau \& Kleinman, 2006; Reis et al., 2004) has suggested that responsiveness is a key determinant in successful relationships. Laurenceau et al. (1998) suggested that selfdisclosure is a vital component in the responsiveness process. Perceived responsiveness needs to be considered in future research comparing $\mathrm{CMC}$ and $\mathrm{FtF}$ interactions to appropriately account for differences between the communication mediums' effects on numerous relationship dimensions (e.g. relationship quality, satisfaction, etc.).

An overlooked aspect in the CMC literature is the novelty of the communication medium. McKenna and Bargh (1999) stated that "the internet is not 'yet' a vital utility such as a telephone" (p. 250). A decade later, Tufekei (2010) reported that hundreds of millions of ordinary individuals interact online where they are no longer anonymous as was the case in early $\mathrm{CMC}$ environments. Along with the frequency of internet use are the perceptions that society holds of CMC. Early research on CMC claimed it as an 
impoverished social experience (Bargh \& McKenna, 2004). The negative perceptions of new forms of communication appear consistently and are concerned with the weakening of communication. I believe the negative perceptions that were once held of $\mathrm{CMC}$ are largely attributable to its novelty at the time.

Early CMC environments throughout the 1990's were assumed to be largely anonymous. McKenna et al. (2002) examined newsgroup members over a two year time period and found that individuals were motivated to bring their online acquaintances offline and relationships formed online to be successful. Boase and Wellman (2005) called for a more holistic account of CMC that places it within the context of all personal relationships instead of viewing CMC relationships as separate from all other social relationships. Recent investigations have shown that individuals use CMC to maintain existing relationships (Jiang et al., 2011) and to interact with individuals already known offline, reducing anonymity's effect on these relationship (Tufekei, 2010). The transition from a largely anonymous CMC environment to an environment that is no longer anonymous has large implications for $\mathrm{CMC}$ relationship research. The novelty of $\mathrm{CMC}$ in the 1990's compared to the current widespread use of CMC by millions of individuals who likely interact with individuals already known involve two different types of interactions and relationships. As CMC becomes a part of everyday life, this medium of communication likely has a different effect on relationships than in the past.

\section{Methodological Implications}

The methodology used in research has a direct influence on how results should be interpreted. Different methodologies will result in different findings. Kim and Dindia (2011) and Nguyen et al. (2011) both provided reviews of the literature regarding self- 
disclosure comparing $\mathrm{CMC}$ and $\mathrm{FtF}$ interactions. What becomes readily apparent when examining these reviews are the inconsistent findings reported throughout the literature. The inconsistent findings reported throughout the literature may be due to the use of different metholodoligies There are two methodological differences that appear to be the most influential; study design and measurement. The designs most commonly used in self-disclosure research are experimental and survey. These two research designs are markedly different on numerous dimensions. Survey designs ask participants to recall post-hoc how much they self-disclose across alternate communication mediums whereas experimental designs manipulate the communication medium through which participants interact and then measures are taken of self-disclosure immediately following the interaction. Treating these two designs as one in the same when comparing FtF interactions with CMC interactions is faulty. Experimental designs measuring selfdisclosure across different communication mediums generally use unacquainted individuals (Mallen et al. 2003; McKenna et al., 2002; Shouten et al. 2009) who are instructed to get acquainted with one another. The differences between survey and experimental designs inevitably results in a different outcome when surveys simply ask individuals how much they disclose online and how much they disclose offline, whereas experiments manipulate the communication medium and ask participants about the level of self-disclosure immediately following the interaction. Participants in the survey studies are likely referring to prior acquaintances and the disclosure within these interactions. Social Penetration Theory states that as relationships develop, the breadth and depth of disclosure increases (Altman \& Taylor, 1973). Under this theoretical framework, measuring disclosure between unacquainted partners and acquainted individuals may be 
measuring two different levels of disclosure. The designs used in studies need to be taken into account when comparing inconsistent findings.

The measurements used in self-disclosure literature are another consideration that needs to be accounted for when interpreting results. Self-disclosure studies either use actual self-disclosure measures or perceived self-disclosure measures. Actual selfdisclosure measures generally involve a content analysis of the interaction, whereas perceived self-disclosure measures are generally self-report measures taken following the interaction. The difference between actual and perceived self-disclosure poses a few problems when comparing online and offline self-disclosure studies. Actual selfdisclosure may be higher when participants interact via CMC due to a text-only environment allowing for disclosure of ASL (age, sex, location) to be counted as actual disclosure. As mentioned previously, individuals may not perceive this information to be self-disclosure and therefore perceive higher levels of self-disclosure via FtF or audio/video CMC interactions when compared to text-only $\mathrm{CMC}$ interactions. I argue that actual self-disclosure and perceived self-disclosure are measuring two different aspects of disclosure. Actual self-disclosure may be defined as the revelation of personal information, but not all personal information revealed may be perceived as selfdisclosure. The current study employed a perceived self-disclosure measure and found FtF and audio/video CMC interactions leading to higher levels than text-only CMC. Because no actual disclosure was recorded, it becomes possible that if actual disclosure had been measured different results may have emerged.

Definitional concerns regarding what is considered self-disclosure also become important when comparing online and offline self-disclosure. Should content analyses of 
text-only interactions define ASL disclosures as self-disclosures when these same disclosures would not occur in a FtF environment due to nonverbal and contextual cues being available and revealing ASL without actual verbal disclosure? It would seem appropriate to deem nonverbal expressions as self-disclosure when analyzing FtF interactions if ASL disclosures are included within text-only CMC environments. The main point regarding methodological issues is that they need to be considered and examined more stringently when comparing online and offline self-disclosure.

\section{Theoretical Implications}

Nguyen et al. (2011) provide a brief overview of five theories that predict differences between FtF and CMC interactions. The social identity model of deindividuation (SIDE), hyperpersonal theory, reduced cues theory (RCT), social information processing theory (SIP), and media richness theory (MRT) are discussed in their review. The SIDE model makes the assumption that disclosure is context-specific and reliant on social norms. The hyperpersonal theory assumes that, due to the lack of cues via $\mathrm{CMC}$, individuals will create exaggerated and idealized perceptions of their interaction partners. RCT and SIP share similarities in the fact that both predict the absence of cues will result in higher levels of disclosure via $\mathrm{CMC}$, although for different reasons. RCT views the absence of cues as a reduced influence of social norms on behavior resulting in disinhibition where individuals will freely disclose more information. On the other hand, SIP views the absence of cues resulting in higher levels of self-disclosure via CMC due to more information requiring disclosure (eg. ASL). MRT and Social Presence Theory are similar to one another. These theories predict FtF interactions to be a richer medium and result in greater social presence (respectively). 
Due to more cues being available in $\mathrm{FtF}$ interactions, these theories propose that more information can be disclosed in FtF interactions.

The current research found no support for the hyperpersonal theory, RCT, or SIP. Participants did not appear to idealize their interaction partners due to reduced cues as evidenced by interaction partners reporting the lowest levels of liking via text-only CMC. Participants in the text-only CMC condition also reported the lowest levels of self and partner disclosure. While it may seem like the current study supports the MRT and Social Presence Theory, I advise that this interpretation be taken with caution. The methodological concerns regarding actual and perceived self-disclosure discussed above need to be considered. Higher levels of actual self-disclosure would fit within the RCT and SIP theoretical frameworks, whereas perceived self-disclosure would appear to fit better under the MRT and Social Presence theoretical frameworks.

The theories reviewed by Nguyen et al. (2011) do not consider the topics of selfdisclosure and rather view disclosure on a continuum from greater levels of disclosure to lower levels of disclosure. The current research asked participants how much their interaction partner disclosed about hobbies/interests, family, major/job, and social life. The results revealed that hobbies/interests and major/job were the main topics of disclosure, and these topics were disclosed at similar levels across communication mediums. Larger differences emerged between communication mediums in regard to whether participants disclosed about their family and social life with participants interacting FtF reporting higher levels of disclosure involving these topics than participants interacting via audio/video or text-only CMC. As CMC becomes more 
prevalent, theories need to be concerned more with the topics that are being disclosed than with the overall level of disclosure.

\section{Strengths and Limitations}

The unstructured nature of the interaction in my study is both a strength and a limitation. The strength involved with the unstructured paradigm is that it mimics reallife get-acquainted interactions and increases the external validity. On the other hand, it becomes a limitation as there is no way to control for what is being discussed during the interactions, and therefore reduces the internal validity. Norton, Frost, and Ariely (2007) and Reis et al. (2011) have had an ongoing commentary on this issue. Reis et al. (2011) argue that while internally valid designs allow for much more control, they do not represent live interaction adequately. Norton, Frost, and Ariely (2011) responded by stating Reis et al. (2011) employed a paradigm far different from everyday interactions. Another limitation of the current study is the environment in which participants interacted. The environment was vastly different from an everyday get-acquainted interaction that takes place in a coffee shop or at the library. Participants were in the Psychology laboratory participating for extra credit or course credit. A more natural environment may reveal different results.

Another limitation is that all participants were students at the same medium sized Midwestern university. This results in interaction partners already having a common ground to stand on when getting acquainted. This makes the results applicable to college students getting acquainted in the university setting, but may not generalize to getting acquainted in other contexts. Also, as mentioned above, there was no way to examine what participants were actually talking about, and was only addressed by asking 
participants how much they felt their interaction partner disclosed about certain topics. No instructions were given as to what the participants were to discuss and may have resulted in discussions far removed from getting to know one another. This approach was taken to make the interactions as natural as possible, but limits the conclusions that can be drawn without first taking the unstructured nature of the interaction into consideration due to the lack of control the experimenters had on what was being discussed.

A final limitation was the use of individual-level analyses. With individual analyses, there is no way to control for or determine partner effects which may influence the results. Further examination may reveal that interaction partners have a larger effect on one another when interacting FtF compared to text-only CMC. Dyadic analyses allow for tests of these effects and may reveal findings not examined with the current study.

\section{Future Directions}

Future research may want to analyze the differences in the topics of disclosure between communication mediums. The current research found differences between communication mediums regarding the topics of disclosure. The communication medium through which social interactions take place may not be what is leading to more or less overall disclosure, but rather more or less disclosure of certain topics. Future research may want to perform content analyses on the topics that are being disclosed. The current research employed participants' perceptions of the topics of disclosure, but it becomes possible that individuals interacting FtF may perceive more disclosure on certain topics than are actually being discussed. In contrast to Jiang et al.'s (2011) conclusion that CMC intensifies the disclosure-intimacy link, the current research found FtF interactions leading to more intimate topics of disclosure (family and social life). Jiang et al. (2011) 
manipulated the level of disclosure (high vs. low) by having a confederate interact with the participants to compile a list of tips for incoming freshman to survive college. Jiang et al.'s (2011) research paradigm is very different from the paradigm employed in the current research. Topics of disclosure may be more intimate via FtF interactions due to the increased number of nonverbal and contextual cues guiding the interaction. Future research should begin to examine the topics of disclosure rather than overall level of disclosure when comparing online and offline communication mediums due to the differences that were revealed in the current research.

Participants were all from the same university, and future research should examine getting acquainted interactions with students from different universities. By utilizing the affordances of $\mathrm{CMC}$ this becomes possible and may reveal fascinating differences by having individuals from different universities interact, hence reducing the level of similarity involved in attending the same university. The topics of disclosure are likely to be different when interacting with an individual from a different university or area of the country. Regarding the topics of disclosure, future research may benefit from manipulating the topics of disclosure. Topic manipulation can be done on numerous levels. Researchers could instruct participants to disclose positive or negative information. The topics of disclosure could also be manipulated by instructing participants to disclose information about family, major/job, hobbies/interests, or social life. By examining the topics of disclosure, many questions could be addressed as to the attributes of $\mathrm{CMC}$ and $\mathrm{FtF}$ interactions that influence social relationships.

$\mathrm{CMC}$ research should begin to explore perceived responsiveness to a greater extent than past research comparing FtF and CMC interactions has. The current research 
suggests that responsiveness is the most influential factor in forming social relationships and liking one's interaction partner. Close relationship research has begun to focus on perceived responsiveness as a vital component in functioning relationships (Clark \& Lemay, 2010). CMC research would benefit by focusing more on responsiveness to reveal unexplored differences between $\mathrm{FtF}$ and $\mathrm{CMC}$ interactions.

\section{Conclusion}

After examining prior literature and the results of the current study, I conclude that $\mathrm{FtF}$ and $\mathrm{CMC}$ interactions can no longer be compared on such broad dimensions that assume $\mathrm{CMC}$ and FtF to be distinctly different forms of communication. Different methodologies and measurements appear to lead to inconsistent findings. Researchers may find value in focusing more on the variables being measured and how they are measured than on differences between communication mediums alone. The current research found differences in the topics disclosed across communication mediums with participants interacting $\mathrm{FtF}$ discussing family and social life more than audio/video $\mathrm{CMC}$ and text-only CMC. As broad variables are examined at a more intricate level, a better understanding of the communication medium's effect on social relationships will likely emerge. The current research suggests that $\mathrm{FtF}$ interactions appear to be the best communication medium in which to get acquainted with another individual. FtF interactions and audio/video CMC interactions were very similar to one another with text-only CMC interactions being the outlier. 


\section{REFERENCES}

Altman, I., \& Taylor, D. A. (1973). Social penetration: The development of interpersonal relationships. New York: Holt, Rinehart \& Winston.

Back, M. D., Stopfer, J. M., Vazire, S., Gaddis, S., Schmukle, S. C., Egloff, B., \& Gosling, S. D. (2010). Facebook profiles reflect actual personality, not selfidealization. Psychological Science, 21, 372-374.

Bargh, J. A., McKenna, K. Y. A., \& Fitzsimons, G. M. (2002). Can you see the real me? Activation and expression of the "true self" on the internet. Journal of Social Issues, 58, 33-48.

Bargh, J. A., \& McKenna, K. Y. A. (2004). The internet and social life. Annual Review of Psychology, 55, 573-590.

Collins, N. L., \& Miller, L. C. (1994). Self-disclosure and liking: A meta-analytic review. Psychological Bulletin, 116, 457-475.

Correa, T., Hinsley, A. W., \& Gil de Zuniga, H. (2010). Who interacts on the web? The intersection of users' personality and social media use. Computers in Human Behavior, 26, 247-253.

Davis, M. H., \& Franzoi, S. L. (1987). Private self-consciousness and self-disclosure. In V. J. Derlega \& J. H. Berg (Eds.), Self-disclosure: Theory, Research, and Therapy (pp. 59-78). New York, NY: Plenum Press.

Fenigstein, A. (1979). Self-consciousness, self-attention, and social interaction. Journal of Personality and Social Psychology, 37, 75-86.

Greene, K., Derlega,V. L.,\& Mathews, A. (2006). Self-disclosure in personal relationships. In A.Vangelisti \&D. Perlman (Eds.), Cambridge handbook of personal relationships. Cambridge, UK: Cambridge University Press, pp. 12681328.

Jiang, L. C., Bazarova, N. N., \& Hancock, J. T. (2010). The disclosure-intimacy link in computer-mediated communication: An attributional extension of the hyperpersonal model. Human Communication Research, 37, 58-77. 
Joinson, A. N. (2001). Self-disclosure in computer-mediated communication: The role of self-awareness and visual anonymity. European Journal of Social Psychology, 31, 177-192.

Kenny, D. A., Kashy, D. A., \& Cook, W. L. (2006). Dyadic Data Analysis. NY: The Guilford Press.

Kim, J. \& Dindia, K. (2011). Online self-disclosure: A review of research. In K. B. Wright \& L. M. Webb (Eds.), Computer-Mediated Communication in Personal Relationships (pp. 156-180). NY: Peter Lang Publishing Inc.

Laurenceau, J. P., Barrett, L. F., \& Pietromonaco, P. R. (1998). Intimacy as an interpersonal process: The importance of self-disclosure, partner disclosure, and perceived partner responsiveness in interpersonal exchanges. Journal of Personality and Social Psychology, 5, 1238-1251.

Laurenceau, J. P. \& Kleinman, B. M. (2006). Intimacy in personal relationships. In A. L. Vangelisti \& D. Perlman (Eds.), The Cambridge Handbook of Personal Relationships (pp.637-653). NY: Cambridge University Press.

Mallen, M. J., Day, S. X., \& Green, M. A. (2003). Online versus face-to-face conversations: An examination of relational and discourse variables. Psychotherapy: Theory, Research,Practice, Training, 40, 155-163.

Matheson, K., \& Zanna, M. P. (1988). The impact of computer-mediated communication on self-awareness. Computers in Human Behavior, 4, 221-233.

McKenna, K. Y. A., \& Bargh, J. A. (1999). Causes and consequences of social interaction on the internet: A conceptual framework. Media Psychology, 1, 249269.

McKenna, K. Y. A., \& Bargh, J. A. (2000). Plan 9 from cyberspace: The implications of the internet for personality and social psychology. Personality and Social Psychology Review, 4, 57-75.

McKenna, K. Y. A., Green, A. S., \& Gleason, M. E. J. (2002). Relationship formation on the internet: What's the big attraction? Journal of Social Issues, 58, 9-31.

Mead, G. H. (1934). Mind, self, and society. Chicago: University of Chicago Press.

Mesch, G., \& Talmud, I. (2006). The quality of online and offline relationships: The role of multiplexity and duration of social relationships. The Information Society, 22, 137-148. 
Montoya, R. M., \& Insko, C. A. (2008). Toward a more complete understanding of the reciprocity of liking effect. European Journal of Social Psychology, 38, 477-498.

Nguyen, M., Bin, Y. S., \& Campbell, A. (2011). Comparing online and offline selfdisclosure: A systematic review. Cyberpsychology, Behavior, and Social Networking, 0(0), 1-9. doi: 10.1089/cyber.2011.0277.

Norton, M. I., Frost, J. H., \& Ariely, D. (2007). Less is more: The lure of ambiguity, or why familiarity breeds contempt. Journal of Personality and Social Psychology, 92, 97-105.

Norton, M. I., Frost, J. H., \& Ariely, D. (2011). Does familiarity breed contempt or liking? Comment on Reis, Maniaci, Caprariello, Eastwick, and Finkel (2011). Journal of Personality and Social Psychology, 101(3), 571-574.

Okdie, B. M., Guadagno, R. E., Bernieri, F. J., Geers, A. L., \& Mclarney-Vesotski, A. R. (2011). Getting to know you: Face-to-face versus online interactions. Computers in Human Behavior, 27, 153-159.

Parks, M. R., \& Floyd, K. (1996). Making friends in cyberspace. Journal of Communication, 46, 80-96.

Parks, M., \& Roberts, L. (1998). Making MOOsic: The development of personal relationships online and a comparison to their off-line counterparts. Journal of Social and Personal Relationships, 15, 517-537.

Peter, J., \& Valkenburg, P. M. (2006). Individual differences in perceptions of internet communication. European Journal of Communication, 21, 213-226.

Ramirez, A., \& Burgoon, J. K. (2004). The effects of interactivity on initial interactions: The influence of information valence and modality and information richness on computer-mediated interaction. Communication Monographs, 71, 422-447.

Ramirez, A. \& Zhang, S. (2007). When online meets offline: The effect of modality switching on relational communication. Communication Monographs, 74, 287310.

Reis, H. T., Clark, M. S., \& Holmes, J. G. (2004). Perceived partner responsiveness as an organizing construct in the study of intimacy and closeness. In D. J. Mashek \& A. P. Aron (Eds.), Handbook of closeness and intimacy (pp. 201-225). Mahwah, NJ: Erlbaum.

Reis, H. T., Maniaci, M. R., Caprariello, P. A., Eastwick, P. W., \& Finkel, E. J. (2011). Familiarity does indeed promote attraction in live interaction. Journal of Personality and Social Psychology, 101(3), 557-570. 
Reis, H. T., Maniaci, M. R., Caprariello, P. A., Eastwick, P. W., \& Finkel, E. J. (2011). In live interaction, does familiarity promote attraction or contempt? Reply to Norton, Frost, and Ariely (2011). Journal of Personality and Social Psychology, 101(3), 575-578.

Reno. R. R., \& Kenny, D. A. (1992). Effects of self-consciousness and social anxiety on self-disclosure among unacquainted individuals: An application of the social relations model. Journal of Personality, 60, 79-94.

Sherman, R. C. (2003). The mind's eye in cyberspace: Online perceptions of self and others. In G. Riva \& C. Galimberti (Eds.), Towards CyberPsychology: Mind, Cognitions and Society in the Internet Age (53-72). Amsterdam: IOS Press.

Schouten, A., Valkenburg, P., \& Peter, J. (2009). An experimental test of processes underlying self-disclosure in computer-mediated communication. Cyberpsychology: Journal of Psychosocial Research on Cyberspace, 3(2), article 1.

Sprecher, S., Treger, S, \& Wondra, J. D. (in press). Effects of self-disclosure role on liking, closeness, and other impressions in get-acquainted interactions. Journal of Social and Personal Relationships, (in press). doi: 10.1177/0265407512459033

Sternberg, R. J. (1988). The triangle of love: Intimacy, passion, and commitment. New York: Basic Books.

Tidwell, L. C., \& Walther, J. B. (2002). Computer-mediated communication effects on disclosure, impressions, and interpersonal evaluations: Getting to know one another a bit at a time. Human Communication Research, 28, 317-348.

Tufekei, Z. (2010). Who acquires friends through social media and why? "Rich get richer" versus "seek and ye shall find." In Proceedings of the $4^{\text {th }}$ International AAAI Conference on Weblogs and Social Media (ICWSM, 2010). AAAI Press. Forthcoming.

Walther, J. B. (2007). Selective self-presentation in computer-mediated communication: Hyperpersonal dimensions of technology, language, and cognition. Computers in Human Behavior, 23, 2538-2557.

Whitty, M. T. (2008). Liberating or debilitating? An examination of romantic relationships, sexual relationships and friendships on the net. Computers in Human Behavior, 24, 1837-1850.

Yao, M. Z., \& Flanagin, A. J. (2006). A self-awareness approach to computer-mediated communication. Computers in Human Behavior, 22, 518-544. 
Zhao, S. (2006). Do internet users have more social ties? A call for differentiated analyses of internet use. Journal of Computer-Mediated Communication, 11, 844862. 
Table 1

Descriptive Statistics and Correlations Among Dependent Measures

\begin{tabular}{|c|c|c|c|c|c|c|c|}
\hline & & $\begin{array}{l}\text { Mean } \\
(\mathrm{SD})\end{array}$ & 1 & 2 & 3 & 4 & 5 \\
\hline 1) & Self-Disclosure & $\begin{array}{l}4.90 \\
(.95)\end{array}$ & - & & & & \\
\hline 2) & $\begin{array}{l}\text { Partner Self- } \\
\text { Disclosure }\end{array}$ & $\begin{array}{l}4.90 \\
(1.06)\end{array}$ & $.74 * * *$ & - & & & \\
\hline 3) & Responsiveness & $\begin{array}{l}3.55 \\
(1.08)\end{array}$ & $.60 * * *$ & $.58 * * *$ & - & & \\
\hline 4) & Liking & $\begin{array}{l}4.69 \\
(1.06)\end{array}$ & $.52 * * *$ & $.56 * * *$ & $.67 * * *$ & - & \\
\hline 5) & Private SA & $\begin{array}{l}2.52 \\
(.78)\end{array}$ & $.22 * *$ & $.18^{*}$ & $.17^{*}$ & .09 & - \\
\hline
\end{tabular}


Table 2

Means, Standard Deviations, and Effect Sizes for Perceived Self-Disclosure, Perceived Partner Self-Disclosure, Perceived Partner Responsiveness, and Private Self-Awareness

\begin{tabular}{lllll}
\hline & FtF & Audio/Video & Text-Only & Partial $\eta^{2}$ \\
\hline Self-Disclosure & $5.15(.98)$ & $5.24(.78)$ & $4.37(.82)$ & .18 \\
Partner Self- & $5.28(.97)$ & $5.13(1.10)$ & $4.33(.88)$ & .16 \\
Disclosure & & & & \\
Responsiveness & $3.88(.99)$ & $3.72(1.15)$ & $3.10(.96)$ & .10 \\
Private SA & $2.37(.80)$ & $2.73(.79)$ & $2.47(.72)$ & .04
\end{tabular}

Wilks' $\Lambda=.76, F(8,272)=5.03, p<.001$ 
Table 3

Multiple Regression on Liking with Self-Disclosure, Partner Disclosure, and Responsiveness as Predictors

\begin{tabular}{lllll}
\hline & $b$ & SE & $\beta$ & $t$-test \\
\hline Self-Disclosure & .03 & .12 & .03 & .29 \\
Partner Self- & .25 & .09 & .25 & $2.65^{*}$ \\
Disclosure & & & & \\
Responsiveness & .49 & .08 & .50 & $6.43 * *$ \\
\hline$* p<.01 ; * * p<.001$ & & & &
\end{tabular}


Table 4

Multiple Regression on Liking with Self-Disclosure, Partner Disclosure, and Responsiveness as Predictors for FtF, Audio/Video, and Text-Only

Conditions

\begin{tabular}{|c|c|c|c|c|c|}
\hline & $R^{2}$ & $b$ & $\mathrm{SE}$ & $\beta$ & $t$-test \\
\hline $\mathrm{FtF}$ & $.56 * * *$ & & & & \\
\hline Self-Disclosure & & .22 & .19 & .17 & 1.14 \\
\hline Partner Self- & & .11 & .18 & .09 & .63 \\
\hline \multicolumn{6}{|l|}{ Disclosure } \\
\hline Responsiveness & & .18 & .04 & .58 & $4.48 * * *$ \\
\hline Audio/Video & $.45 * * *$ & & & & \\
\hline Self-Disclosure & & -.06 & .32 & -.04 & -.20 \\
\hline Partner Self- & & .33 & .21 & .26 & 1.59 \\
\hline \multicolumn{6}{|l|}{ Disclosure } \\
\hline Responsiveness & & .16 & .04 & .53 & $3.68 * *$ \\
\hline Text-Only & $.42 * * *$ & & & & \\
\hline Self-Disclosure & & -.18 & .30 & -.10 & -.58 \\
\hline Partner Self- & & .73 & .32 & .45 & $2.27 *$ \\
\hline \multicolumn{6}{|l|}{ Disclosure } \\
\hline Responsiveness & & .13 & .05 & .34 & $2.36^{*}$ \\
\hline
\end{tabular}


Table 5

Means and Standard Deviations for Disclosure Topics

\begin{tabular}{lllll}
\hline & FtF & Audio/Video & Text-Only & Total \\
\hline Hobbies or & $4.04(1.78)$ & $4.20(1.41)$ & $3.68(1.68)$ & $3.96(1.64)$ \\
\multicolumn{1}{c}{ Interests } & & & & \\
Family & $3.20(2.05)$ & $2.70(1.40)$ & $1.52(1.15)$ & $2.44(1.71)$ \\
Major or Job & $5.89(1.06)$ & $5.35(1.37)$ & $5.38(1.47)$ & $5.54(1.33)$ \\
Social Life & $4.13(1.46)$ & $3.96(1.66)$ & $2.66(1.30)$ & $3.56(1.61)$ \\
\hline
\end{tabular}

Wilks' $\Lambda=.72, F(8,272)=6.20, p<.001$ 
Table 6

Means and Standard Deviations for Reported Difficulty in Self-Disclosure and Responsiveness

\begin{tabular}{lllll}
\hline & FtF & Audio/Video & Text-Only & Total \\
\hline Self-Disclosure & $1.74(.98)$ & $2.52(1.17)$ & $2.26(1.40)$ & $2.18(1.23)$ \\
Responsiveness & $1.54(.96)$ & $2.37(1.08)$ & $1.94(1.32)$ & $1.95(1.18)$ \\
\hline
\end{tabular}

Wilks' $\Lambda=.90, F(4,276)=3.54, p=.008$ 